\title{
Information Representation and Communication Pattern for Unified Management of Software-Defined Networking
}

\author{
Hui $\mathrm{Xu}$, Chunzhi Wang and Hongwei Chen \\ School of Computer Science, Hubei University of Technology, Wuhan, China \\ xuhui@mail.hbut.edu.cn
}

\begin{abstract}
When considering the coexistence of Software-Defined Networking (SDN) and non$S D N$, unified management of SDN calls for study on both the information representation and the communication pattern. This paper first proposes the use of the extension theory in order to formally define management information of SDN in a unified manner. And this paper then introduces the concept of unified manager to the communication pattern for unified management of SDN, and considers application of the queuing theory from the perspective of the basic queue and the queue spaces. Finally, simulation results validate that, proposed approach based on the queuing theory is feasible for unified management of SDN from the viewpoint of the communication pattern.
\end{abstract}

Keywords: Software-Defined Networking, unified management, information representation, communication pattern, queuing theory

\section{Introduction}

As for unified management of Software-Defined Networking (SDN) [1], both the information representation and the communication pattern should be seriously studied, when taking the coexistence of SDN and non-SDN [2] into consideration.

Open Networking Foundation (ONF) that is an organization to promote SDN standardizations, proposes not only the SDN architecture [3-4] but also the OpenFlow management and configuration protocol (OF-CONFIG, the newest version is 1.2 up to now) [5]. Current version 1.2 of OF-CONFIG uses the module companioning with YANG [6] to realize its data model, including type definitions, groupings and main container [5]. However, the coexistence of SDN and non-SDN leads to the difficulty in formally defining management information of SDN by means of unified representations. Additionally, the communication pattern should be considered based on the information representation, in order to study and solve the problem of unified management for SDN.

Our prior work [7] tried to apply the extension theory and the queuing theory to monitor service level parameters of SDN and propose an integrated approach based on both two theories from the formalization point of view, and further work based on the extension theory for threat detection and defense of SDN have also been under research. It seems that, the extension theory promotes the formalization of unified representations for management information of SDN, and the queuing theory prospects a promising way for the management of SDN so as to understand the queuing principles between management applications and the SDN manager. However, more managers are required for the case of unified management integrating SDN with non-SDN, and application of the queuing theory for this case must be seriously studied.

The remainder of this paper is organized as follows. Section 2 discusses issues related to the information representation for unified management of SDN, and proposes the use of the extension theory to formally define management information 
of SDN in a unified manner. Section 3 focuses on the communication pattern for unified management of SDN, introduces the concept of unified manager, and considers the application of the queuing theory, including the basic queue and the queue spaces. Section 4 validates the feasibility of proposed approach based on the queuing theory for unified management of SDN from the viewpoint of the communication pattern. Section 5 concludes this paper.

\section{Information Representation for Unified Management of SDN}

As to information representation for unified management of SDN, the extension theory for Extenics [8] may possibly be utilized to promote formal representations of management information of SDN, and matter-elements are then introduced to formally define management information of SDN in a unified manner.

\subsection{Application of the Extension Theory}

Based on the extension theory, the matter-elements for the information representation in view of unified management of SDN can be formally defined as an ordered three-tuple $M_{i r}=\left(O_{i r}, c_{i r}, v_{i r}\right)$, in which $O_{i r}$ is an object of information representation for unified management of SDN with the characteristic $c_{i r}$ and the corresponding value $v_{i r}$.

Furthermore, the class for this kind of matter-elements is then formally defined as $\left\{M_{i r}\right\}=\left(\left\{O_{i r}\right\}, c_{i r}, V_{i r}\right)$, in which $V_{i r}$ describes the value domain of the characteristic $c_{i r}$ for the set of objects that is $\left\{O_{i r}\right\}$.

Since management information of SDN might be related to each other, thus the matterelements and their composition forms that are the composite-elements are then utilized to formally define the information representation for unified management of SDN.

\subsection{Formal Information Representation based on the Extension Theory}

According to current version 1.2 of OF-CONFIG and its proposed data model with the use of YANG, matter-elements and composite-elements are used to formally describe management information of SDN in a unified manner.

Formula (1) formally defines the OpenFlow capable switch, which relates to the OpenFlow configuration point, the OpenFlow resource and the OpenFlow logical switch, respectively with formalizations as Formula (2), Formula (3) and Formula (4).

$$
\begin{gathered}
M_{c s}=\left[\begin{array}{c}
O_{c s}, i d, v_{i d} \\
\text { config-version, } v_{\text {config-version }} \\
\text { configuration-point } s,\left\{M_{c p}\right\} \\
\text { resources, }\left\{M_{r}\right\} \\
\log \text { ical }- \text { switches },\left\{M_{l s}\right\}
\end{array}\right] \\
M_{c p}=\left[\begin{array}{c}
O_{c p}, \text { id }, v_{i d} \\
\text { url }, v_{\text {url }} \\
\text { protocal }, v_{\text {protocol }} \\
\text { traffic }, v_{\text {traffic }}
\end{array}\right]
\end{gathered}
$$




$$
\begin{gathered}
M_{r}=\left[O_{r}, \text { resource }-i d, v_{\text {rid }}\right] \\
M_{l s}=\left[\begin{array}{c}
O_{l s}, \text { id }, v_{\text {id }} \\
\text { datapath }-i d, v_{\text {did }} \\
\text { enabled }, v_{\text {enabled }} \\
\text { check - controller - certificate }, v_{c c c} \\
\text { lost - connection - behavior }, v_{l c b} \\
\text { reources },\left\{M_{r}\right\} \\
\text { capabilities },\left\{M_{l s c}\right\} \\
\text { controllers },\left\{M_{o f c}\right\}
\end{array}\right]
\end{gathered}
$$

As indicated in Formula (4), the OpenFlow logical switch not only relates to the OpenFlow resource, but also associates with the logical switch capability and the OpenFlow controller, which can also be respectively formalized as $M_{l s c}$ and $M_{o f c}$ in the same way that using matter-elements and composite-elements.

To sum up, the extension theory promotes the information representation for unified management of SDN, by applying matter-elements and composite-elements to formally define management information of SDN in a unified manner.

\section{Communication Pattern for Unified Management of SDN}

In order to study and solve the problem of unified management for SDN, the communication pattern should be further considered based on the information representation using matter-elements and composite-elements. The queuing theory then prospects a promising way for the study on the communication pattern of unified management for SDN.

\subsection{Application of the Queuing Theory}

The concept of unified managers is first introduced to deal with the service requests of network management from various applications for both SDN and nonSDN, in which these requests are random, the number of unified managers is limited, and the response time of unified managers is also random. Thus in this case, the problem of unified management can be seen as service requesting and responding between the applications and the unified managers, and the phenomenon of queuing may possibly exist for this process. Therefore, the queuing theory [9] is then applied to unified management of SDN, in order to solve the queuing problem between the applications and the unified managers.

As to the basic queue of unified management for SDN, unified managers are allocated so as to deal with the service requests of network management from various applications for both SDN and non-SDN. Suppose that

a) The service request of network management from various applications conforms to a negative exponential distribution with parameter $\alpha$.

b) The serving time of unified managers for requests from various applications conforms to a negative exponential distribution with parameter $\beta$.

c) According to the principle of First-Come-First-Serve (FCFS), $n$ unified managers are allocated to deal with the service requests of network management from various applications for both SDN and non-SDN.

d) The capacity of the queue between the applications and the unified managers is $K(K \geq n+1)$. 
In this case, a $M / M / n / K-F C F S$ queue, in which $M$ means the exponential distribution, is applied to study the queuing problem between the applications and the unified managers. As for this queue, the number of applications requesting services of network management for both SDN and non-SDN forms a birth-anddeath process. Figure 1 shows the transition graph of this $M / M / n / K-F C F S$ queue between the applications and the unified managers for both SDN and non-SDN.

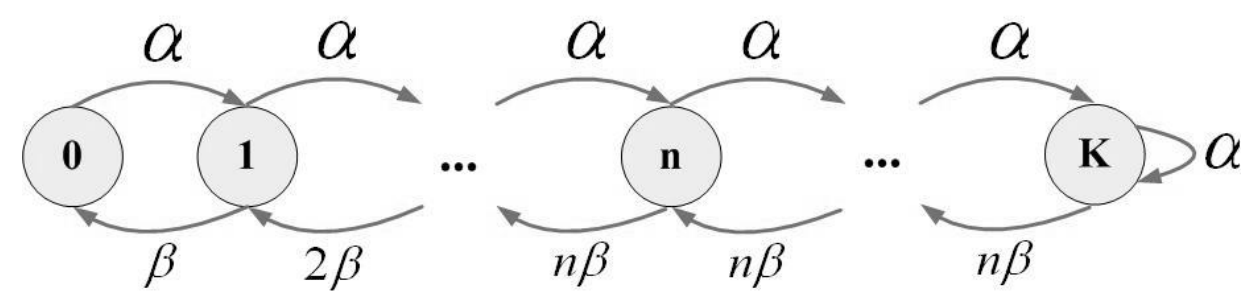

Figure 1. Transition Graph of the $M / M / n / K-F C F S$ Queue between the Applications and the Unified Managers for Both SDN and Non-SDN

Furthermore, as for this $M / M / n / K-F C F S$ queue for the queuing problem between the applications and the unified managers, $p_{u}=P\{U=u\}(u=0,1,2, \ldots)$ is introduced to describe the distribution for the number of applications requesting services of network management for both SDN and non-SDN.

Assume that $\lambda=\frac{\alpha}{\beta}$ and $\lambda_{n}=\frac{\alpha}{n \beta}$, then according to the queuing theory, the performance measurements in this $M / M / n / K-F C F S$ queue for the queuing problem between the applications and the unified managers can be studied as follows.

a) The possibility of idle state for the unified managers in SDN is

$$
p_{0}=\left\{\begin{array}{l}
{\left[\sum_{u=0}^{n-1} \frac{\lambda^{u}}{u !}+\frac{\lambda^{n}\left(1-\lambda_{n}^{K-n+1}\right)}{n !\left(1-\lambda_{n}\right)}\right]^{-1}, \lambda_{n} \neq 1} \\
{\left[\sum_{u=0}^{n-1} \frac{\lambda^{u}}{u !}+\frac{\lambda^{n}}{n !}(K-n+1)\right]^{-1}, \lambda_{n}=1}
\end{array}\right.
$$

b) Based on Formula (5), the possibility distribution for the number of applications requesting the services of SDN management is

$$
p_{u}=\left\{\begin{array}{l}
\frac{\lambda^{u}}{u !} p_{0}, 0 \leq u<n \\
\frac{\lambda^{u}}{n ! n^{u-n}} p_{0}, n \leq u \leq K
\end{array}\right.
$$

c) The average queue length of this $M / M / n / K-F C F S$ queue for the queuing problem between the applications and the unified managers is

$$
L_{1}=\left\{\begin{array}{l}
\frac{p_{0} \lambda^{n} \lambda_{n}}{n !\left(1-\lambda_{n}\right)^{2}}\left[1-\lambda_{n}^{K-n+1}-\left(1-\lambda_{n}\right)(K-n+1) \lambda_{n}^{K-n}\right], \lambda_{n} \neq 1 \\
\frac{p_{0} \lambda^{n}(K-n)(K-n+1)}{2 n !}, \lambda_{n}=1
\end{array}\right.
$$


d) Based on Formula (5) and Formula (7), the average length of this $M / M / n / K-F C F S$ queue for the queuing problem between the applications and the unified managers is

$$
L_{2}=L_{1}+n+p_{0} \sum_{u=0}^{n-1} \frac{(u-n) \lambda^{u}}{u !}
$$

At the same time, there is

$$
L_{2}=L_{1}+\lambda\left(1-p_{K}\right)
$$

e) According to Little Formula in the queuing theory, the average staying time of this $M / M / n / K-F C F S$ queue for the queuing problem between the applications and the unified managers is

$$
T_{3}=\frac{L_{2}}{\alpha\left(1-p_{K}\right)}
$$

f) According to Little Formula in the queuing theory, the average waiting time of this $M / M / n / K-F C F S$ queue for the queuing problem between the applications and the unified managers is

$$
T_{4}=\frac{L_{1}}{\alpha\left(1-p_{K}\right)}
$$

At the same time, there is

$$
T_{4}=T_{3}-\frac{1}{\beta}
$$

\subsection{Queue Spaces for Unified Management of SDN}

As for unified management of SDN, in order to avoid long-time queue waiting for service requests from applications because of an insufficient number of unified managers, two queue spaces, which are the high-priority queue space and the public queue space, are introduced to the basic queue of unified management for SDN.

Suppose that $A(t)$ is the state of the high-priority queue space at time $t$, in which if this space is available, then $A(t)=1$, else $A(t)=0$. Similarly, assume that $B(t)$ is the state of the public queue space at time $t$, in which if this space is available, then $B(t)=1$, else $B(t)=0$.

Furthermore, suppose that a service request with high-priority from the application arrives according to a Poisson process with the intensity $\alpha_{x}$, and when $A(t)=1$ and $B(t)=1$, this request randomly enters into the high-priority queue space or the public queue space.

Additionally, suppose that a common service request from the application arrives according to a Poisson process with the intensity $\alpha_{y}$, and when $B(t)=1$, this request enters into the public queue space. It is also assumed that, the queuing rule adopts a loss style, that is to say, when the queue space chosen by the service request from the application is occupied, this request will be abandoned.

Thus in this case, from the viewpoint of the high-priority queue space and the public queue space to study on unified management of $\operatorname{SDN},(A(t), B(t))$ is a 
Markov process. Figure 2 then provides the transition graph of this Markov process associated to the high-priority queue space and the public queue space for unified management of SDN.

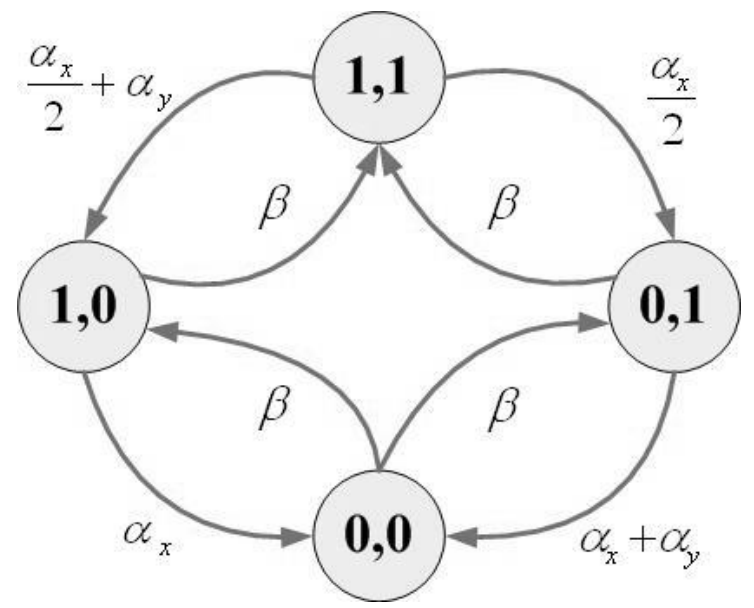

Figure 2. Transition Graph of the Markov Process Associated to the High-Priority Queue Space and the Public Queue Space for Unified Management of SDN

\section{Simulation Results}

In order to validate the feasibility of proposed $M / M / n / K-F C F S$ queue for unified management of SDN from the viewpoint of the communication pattern, $\alpha$ is set to $2, \beta$ is set to 0.5 and $K$ is set to 10 .

Figure 3-6 respectively show the changes of the average queue length, the average length, the average staying time and the average waiting time for this queue when the number of unified managers for SDN increases from 2 to 9.

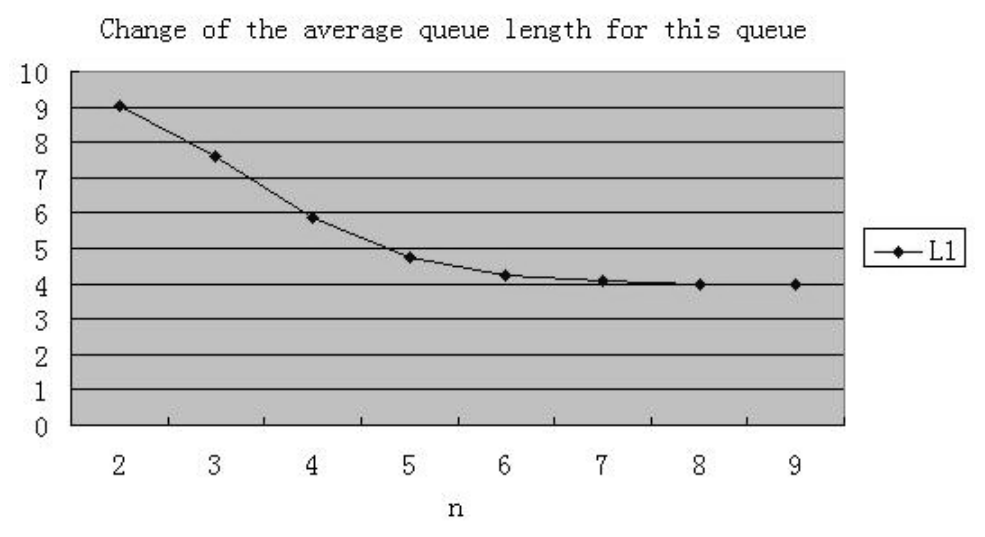

Figure 3. Change of the Average Queue Length for this Queue when the Number of Unified Managers for SDN Increases from 2 to 9 
Change of the average length for this queue

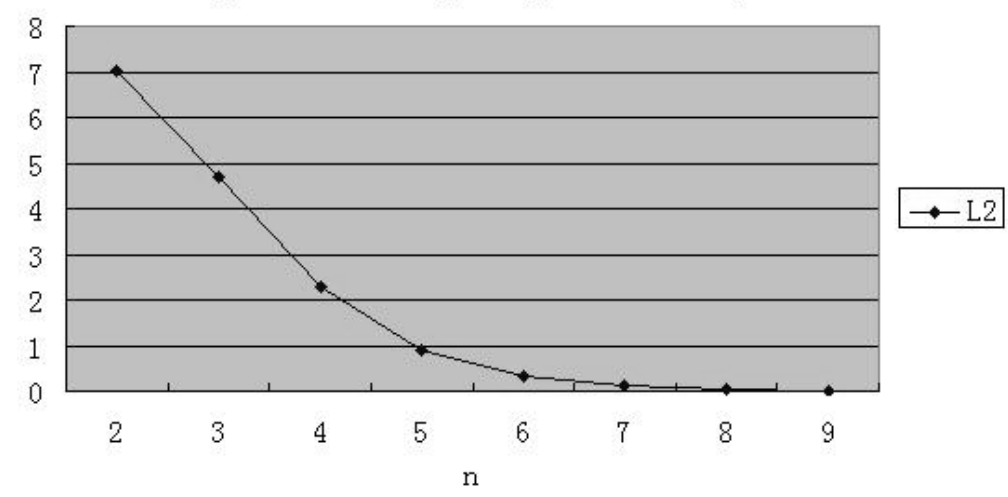

Figure 4. Change of the Average Length for this Queue when the Number of Unified Managers for SDN Increases from 2 to 9

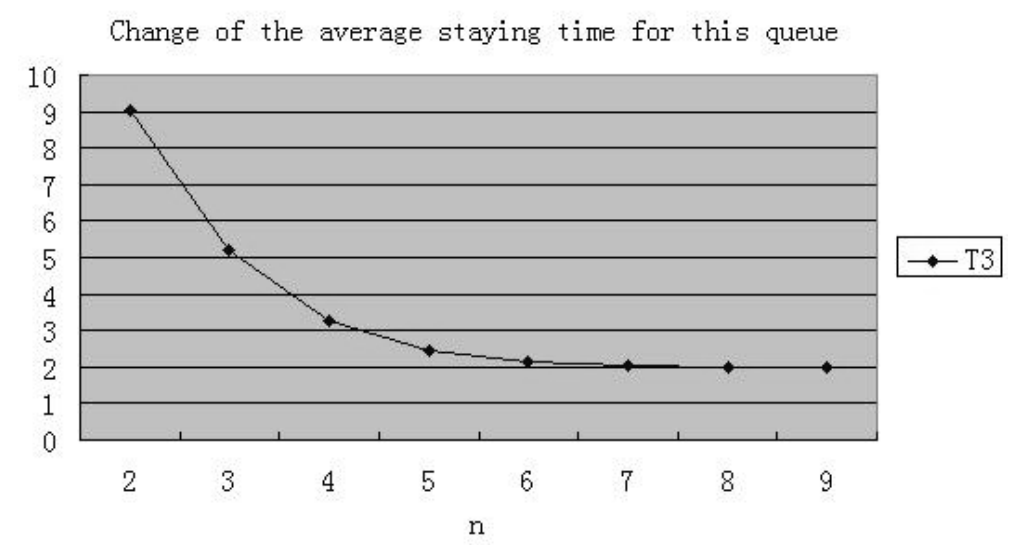

Figure 5. Change of the Average Staying Time for this Queue when the Number of Unified Managers for SDN Increases from 2 to 9

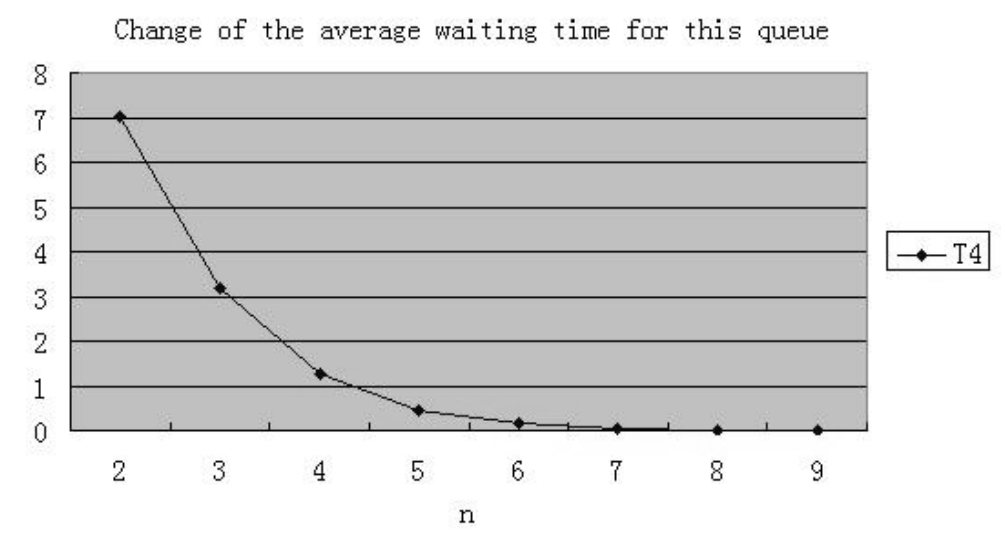

Figure 6. Change of the Average Waiting Time for this Queue when theNumber of Unified Managers for SDN Increases from 2 to 9 
As respectively indicated in Figure 3-6, when the number of unified managers for SDN increases from 2 to 9, the average queue length, the average length, the average staying time and the average waiting time for this queue all reduce significantly at the beginning and tend to reduce at a relatively slower rate in the later stage.

In summary, simulation results validate that, proposed approach based on the queuing theory is feasible for unified management of SDN from the communication pattern point of view.

\section{Conclusions}

The main contribution of this paper is to focus on both the information representation and the communication pattern for unified management of SDN due to the coexistence of SDN and non-SDN, propose the use of the extension theory so as to formally define management information of SDN by means of unified representations, and considers the application of the queuing theory to the communication pattern for unified management of SDN, including the basic queue and the queue spaces. Simulation results validate the feasibility of proposed approach based on the queuing theory for unified management of SDN from the communication pattern point of view.

\section{Acknowledgements}

This work has been supported by the Emergency Management Program for National Natural Science Foundation of China (No. 61440024), the Provincial Teaching Reform Research Project of Education Department of Hubei Province in China (No. 2012273), the Doctoral Scientific Research Fund from Hubei University of Technology (No. BSQD12029), the National Natural Science Foundation of China for Young Scholars (No. 61202287, No. 41301371) and the General Program for National Natural Science Foundation of China (No. 61170135). The authors would like to thank all project partners for their valuable contributions and feedbacks.

\section{References}

[1] N. Koutsouris, K. Tsagkaris, P. Demestichas, L. Mamatas, S. Clayman and A. Galis, "Managing Software-Driven Networks with a Unified Management Framework", Proceeding of 13th IFIP/IEEE International Symposium on Integrated Network Management, (2013), pp. 1084-1085.

[2] D. Kim, J.M. Gil, G. Wang G and S. Kim, "Integrated SDN and Non-SDN Network Management Approaches for Future Internet Environment”, Lecture Notes in Electrical Engineering, vol. 240, (2013), pp. 529-536.

[3] Open Networking Foundation, "SDN Architecture Issue 1", (2014).

[4] Open Networking Foundation, "SDN Architecture Overview Version 1.1", (2014).

[5] Open Networking Foundation, "OpenFlow Management and Configuration Protocol 1.2 (OF-CONFIG 1.2)”, Available: www.opennetworking.org/technical-communities/areas/specification/1928-of-config, (2016).

[6] M. Bjorklund ed, "YANG - A Data Modeling Language for the Network Configuration Protocol (NETCONF)", RFC6020, (2010).

[7] [7] H. Xu and H.W. Chen, "An Integrated Approach for Monitoring Service Level Parameters of Software-Defined Networking", International Journal of Future Generation Communication and Networking, vol. 8, no. 6, (2015), pp. 197-204.

[8] C.Y. Yang and W. Cai, "Extenics", Science Press, Beijing, (2014), Simplified Chinese version.

[9] D. Gross, J.F. Shortle, J.M. Thompson and C.M. Harris, "Fundamental of Queuing Theory (Fourth Edition)", Wiley Press, (2008). 


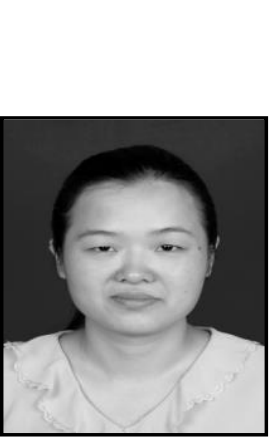

\begin{abstract}
Authors
Hui Xu, She received a bachelor's degree in Computer Science and Technology from Huazhong Normal University, Wuhan, China in 2005, a master's degree in Computer Application Technology from Huazhong Normal University, Wuhan, China in 2008, and a doctor's degree in Radio Physics from Huazhong Normal University, Wuhan, China in 2010. Since 2006, she has been a certified computer system analyst in China. Now, she is an Associate Professor at the School of Computer Science in Hubei University of Technology, Wuhan, China. Currently, her major field of study is network and service management.

Dr. Xu became a Member of Institute of Electrical and Electronics Engineers (IEEE) in 2007, a Member of Association for Computing Machinery (ACM) in 2007 and a Member of China Computer Federation (CCF) in 2008. She has authored or coauthored 1 book and 2 book chapters in the field of network management, about 10 papers published by Chinese journals, more than 10 papers published by international journals, and more than 20 papers published by international conferences. In April 2008, she was awarded by International Association of Engineers (IAENG) for her firstauthored paper presented to 2008 IAENG International Conference on Communication Systems and Applications. Additionally, she was a Session Co-Chair or a Paper Reviewer for 2nd\&3rd\&7th\&8th International Conference on Computer Science and Education (ICCSE 2007\&2008\&2012\&2013), a Session Chair for 1st International Symposium on Electronic Commerce and Security (ISECS 2008), a Paper Reviewer for 4th IEEE Conference on Industrial Electronics and Applications (ICIEA 2009), a Paper Reviewer for 3rd International Conference on Computer and Network Technology (ICCNT 2011), a Paper Reviewer for 32nd Chinese Control Conference (CCC 2013), and a Paper Reviewer for Security and Communication Networks, an international journal published by Wiley Press.
\end{abstract}

Chunzhi Wang, She is a Professor at the School of Computer Science in Hubei University of Technology, Wuhan, China. She is also the Dean of the School of Computer Science in Hubei University of Technology, Wuhan, China. Currently, her major field of study is Software-Defined Networking.

Hongwei Chen, $\mathrm{He}$ is a Professor at the School of Computer Science in Hubei University of Technology, Wuhan, China. Currently, his major field of study is distributed management. 
International Journal of Grid and Distributed Computing Vol. 9, No. 11 (2016) 